\title{
Overlap of the cancer genome atlas and the immune epitope database
}

\author{
SHAIMAA SAIT ${ }^{1}$, TIMOTHY FAWCETT ${ }^{2}$ and GEORGE BLANCK ${ }^{1,3}$ \\ ${ }^{1}$ Department of Molecular Medicine, Morsani College of Medicine, \\ Tampa, FL 33612; ${ }^{2}$ Department of Chemical and Biomedical Engineering, College of Engineering, \\ Research Computing University of South Florida, Tampa, FL 33620; ${ }^{3}$ Immunology Program, \\ Moffitt Cancer Center and Research Institute, Tampa, FL 33612, USA
}

Received August 28, 2015; Accepted May 16, 2016

DOI: $10.3892 / \mathrm{ol} .2016 .4991$

\begin{abstract}
Mutant peptides resulting from cancer drivers or passenger mutations are expected to have the potential to serve as a basis for cancer vaccines. However, a number of parameters regulate vaccine-associated immunogenicity, including the suitability of a peptide for binding to an antigen-presenting molecule or antibody. In order to obtain a basic indication of the prospect of human cancer epitope identification via current database development strategies, an overlap of the mutant Homo sapiens epitopes listed on the Immune Epitope Database (IEDB) and the mutant peptides indicated by The Cancer Genome Atlas (TCGA) somatic mutation database was obtained. No putative TCGA mutant peptides were detected among the 8,890 14-18 amino acid (AA) IEDB peptides available. In total, 3 IEDB mutant epitopes that encompassed a TCGA mutant AA position, but did not overlap the exact position of the TCGA mutant AA, were detected. The results of the present analysis confirm that verification of certain aspects of cancer epitope function can be obtained via the continued and systematic expansion of databases representing human protein epitopes. However, the analysis also indicates that there is relatively limited systematic information available regarding antigen-presenting molecule epitopes and cancer-related mutant peptides.
\end{abstract}

\section{Introduction}

The development of cancer vaccines has become a high priority in the field of cancer treatment. However, there are numerous parameters that affect the success of an immune

Correspondence to: Professor George Blanck, Department of Molecular Medicine, Morsani College of Medicine, 12901 Bruce B Downs Blvd, Tampa, FL 33612, USA

E-mail: gblanck@health.usf.edu

Key words: The Cancer Genome Atlas, Immune Epitope Database, bioinformatics, cancer immunology, immuno-peptidome project, extra-cellular matrix proteins, integrin subunit $\beta 3$, collagen type II $\alpha 1$ response against an antigen, including the binding of a T-cell receptor to a major histocompatibility complex (MHC)-bound antigen and antigen processing. Empirical evaluations of vaccine efficacy parameters are costly and time-consuming. Thus, bioinformatic approaches may provide a useful alternative.

The Immune Epitope Database (IEDB) includes $>35,000$ human peptides known to either bind to human leukocyte antigen (HLA) class I or II, or to have other immune receptor binding properties $(1,2)$. Knowledge of the capacity of a peptide to bind to antigen-presenting molecules could potentially improve the selection of cancer vaccine candidates that are based on mutant peptides, whether these result from cancer drivers or passenger mutations. In addition, sufficient database development may allow for a better understanding of any presumed selection against the binding of cancer peptide neoantigens to MHC molecules as an aspect of cancer development.

The present study focused on searching for overlaps of The Cancer Genome Atlas (TCGA) mutant peptides $(3,4)$ and peptides in the IEDB, in order to discover cancer-related peptides that have the demonstrable capability to bind to $\mathrm{MHC}$ molecules.

\section{Materials and methods}

The overview of the approach is provided in Fig. 1. Supporting online material (SOM) representing each stage of the approach were also used in the present study (http://www.universityseminarassociates.com/Supporting_online_material_for_scholarly _pubs.php) (5). Briefly, human epitopes were downloaded from the IEDB (www.iedb.org) using the following search terms: Epitope, linear epitope; antigen, Homo sapiens (human) (ID: 9606, Homo sapiens); host, humans; assay, all assays; MHC restriction, MHCI and MHCII; disease, any disease. The results comprised $\sim 35,000$ epitopes and were downloaded as an Excel file. Epitopes that ranged between 14-18 amino acids (AAs) in size were used to determine mismatches with the human genome version 19 (hg19) reference genome at genome.ucsc.edu. The nucleotide spans of the mismatches were obtained by using the BLAT search (http://genome.ucsc.edu/cgi-bin/hgBlat?command=start)tosearch 
Table I. Identification of IEDB peptides that overlap the position of a mutant amino acid in the TCGA database.

\begin{tabular}{|c|c|c|c|c|c|c|c|c|}
\hline $\begin{array}{l}\text { Hugo } \\
\text { gene } \\
\text { symbol }\end{array}$ & $\begin{array}{l}\text { Normal } \\
\text { peptide } \\
\text { sequence } \\
\text { (hg19) }\end{array}$ & $\begin{array}{c}\text { IEDB } \\
\text { ID } \\
\text { number }\end{array}$ & $\begin{array}{l}\text { IEDB peptide } \\
\text { (hg19 } \\
\text { mismatched } \\
\text { amino acid in } \\
\text { large type, } \\
\text { bold) }\end{array}$ & $\begin{array}{l}\text { hg19 translation } \\
\text { of TCGA } \\
\text { mutation on } \\
\text { either side of } \\
\text { mutation } \\
\text { position }\end{array}$ & $\begin{array}{c}\text { TCGA } \\
\text { mutation } \\
\text { chromosome } \\
\text { number }\end{array}$ & $\begin{array}{l}\text { TCGA } \\
\text { cancer } \\
\text { dataset }\end{array}$ & $\begin{array}{c}\text { TCGA } \\
\text { nucleotide } \\
\text { number }\end{array}$ & MHC \\
\hline ATP1A2 & $\begin{array}{l}\text { NPREAKACV } \\
\text { VHGSDLK }\end{array}$ & 103447 & $\begin{array}{l}\text { NPRDAKAC } \\
\text { VVHGSDLK }\end{array}$ & $\begin{array}{l}\text { VVHGSDL } \\
\text { ACVVHG } \\
\text { CVVHGS } \\
\text { VVHGSDL }\end{array}$ & $\begin{array}{l}1 \\
1 \\
1 \\
1\end{array}$ & $\begin{array}{l}\text { LUAD/ } \\
\text { LIHC } \\
\text { LIHC } \\
\text { LIHC } \\
\text { LUAD/ } \\
\text { LIHC }\end{array}$ & $\begin{array}{l}160104960 \\
160104952 \\
160104955 \\
160104960\end{array}$ & $\begin{array}{l}\text { HLA- } \\
\text { DQB }\end{array}$ \\
\hline COL2A1 & $\begin{array}{l}\text { GEPGIAGFK } \\
\text { GEQGPKG }\end{array}$ & 107398 & $\begin{array}{l}\text { GKPGIAGFK } \\
\text { GEQGPKG }\end{array}$ & IAGFKG & 12 & READ & 48380651 & $\begin{array}{l}\text { HLA- } \\
\text { DRB1 }\end{array}$ \\
\hline TROVE2 & $\begin{array}{l}\text { LQEMPLTAL } \\
\text { LRNLGKM }\end{array}$ & 118499 & $\begin{array}{l}\text { LQEMPTLA } \\
\text { LLRNLGKM }\end{array}$ & ALLRNL & 1 & SKCM & 193045674 & NA \\
\hline
\end{tabular}

Data were obtained from the supporting online material files by Sait et al (5) (http://www.universityseminarassociates.com/Supporting_online_ material_for_scholarly_pubs.php) using the procedure described in Fig. 1. IEDB, Immune Epitope Database; TCGA, The Cancer Genome Atlas; hg19, human genome version 19; ID, identification; MHC, major histocompatibility complex; ATP1A2, ATPase $\mathrm{Na}^{+} / \mathrm{K}^{+}$transporting subunit $\alpha$ 2; LUAD, lung adenocarcinoma; LIHC, liver hepatocellular carcinoma; READ, rectum adenocarcinoma; SKCM, skin cutaneous melanoma; COL2A1, collagen type II $\alpha$ 1; TROVE2, Telomerase, Ro and Vault domain family member 2; LUAD, Lung Adenocarcinoma; HLA-DQB, major histocompatibility complex, class II, DO $\beta$; HLA-DRB1, major histocompatibility complex, class II, DR $\beta$ 1; NA, not applicable.

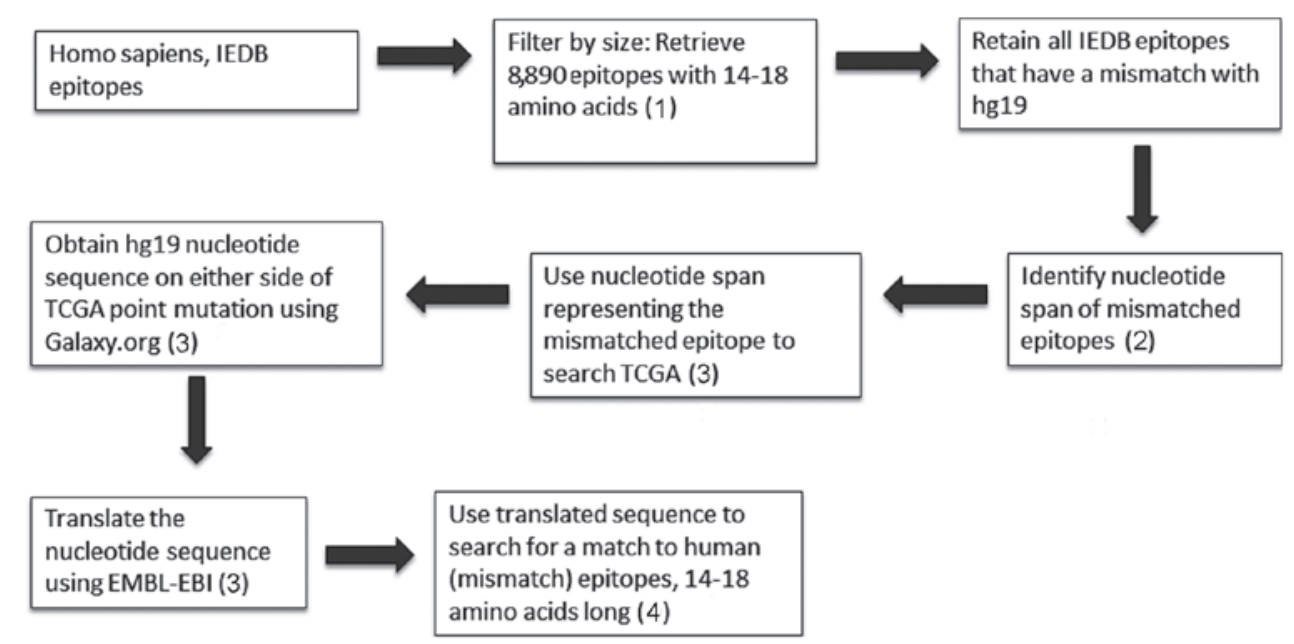

Figure 1. Overview of the procedure used to determine whether any IEDB peptides, which did not match the hg19, matched putative TCGA mutant peptides. The file numbers (1-4) in the figure refer to the supporting online material files by Sait et al (http://www.universityseminarassociates.com/Supporting_online_ material_for_scholarly_pubs.php), which include results and details on each step of the procedure. IEDB, Immune Epitope Database; TCGA, The Cancer Genome Atlas; hg19, human genome version 19; EMBL-EBI, European Molecular Biology Laboratory-European Bioinformatics Institute.

a local database, which was created from the TCGA download portal and consisted of a collection of all TCGA cancer mutations (https://tcga-data.nci.nih.gov/tcga/tcgaDownload.jsp).Therecovered nucleotide positions were extended with hg19 nucleotides using The Extract Genomic DNA tool at https://usegalaxy.org/. The extended regions were then translated into all possible reading frames (including forward and reverse) using the European Molecular Biology Laboratory-European Bioinformatics Institute to generate a database for screening the IEDB 14-18 AA set, in order to verify the IEDB matches and to detect mismatches at the location of the TCGA mutation. All HLA candidates were removed due to overly extensive sequence variation. Gene family members that were originally inaccurately regarded as hg19 mismatches, may be found in the SOM files by Sait et al (5) (Table I).

\section{Results and Discussion}

The present study was required to determine whether detecting an IEDB peptide that had a mismatch at the exact position of 
a TCGA mutant AA was possible. Therefore, a search was performed among the 8,890 IEDB human peptides consisting of 14-18 AAs, with translated AAs on either side of all TCGA point mutations, to check for overlap with an IEDB epitope that had a mismatch with the hg19 version of the reference genome. Since the translations represented exact matches with the hg19 translations, the 8,890 epitopes consisting of 14-18 AA were searched, allowing for one mismatch with the translations used, in order to 'surround' the location of the TCGA mutation. According to this protocol, while the TCGA point mutation-referenced translations overlapped the position of the TCGA mutation, these translations matched hg19 exactly, thus requiring the single mismatch standard for searching the aforementioned 8,890 IEDB epitopes for an exact match.

Numerous IEDB epitopes were identified using this method; however, following the exclusion of IEDB epitopes that did not match the gene of the TCGA mutation, only one IEDB peptide had a non-hg19 AA in the position of the TCGA mutant AA. This IEDB epitope mapped to integrin subunit $\beta 3$ (ITGB3), which is a known ITGB3 single nucleotide polymorphism. The data supporting this finding is presented in SOM file no. 5 of Sait et al (5).

To determine whether the TCGA mutant AA positions overlapped IEDB peptides that contained a mismatch with the hg19 AA sequence, without the TCGA position equaling the precise location of the IEDB mismatched AAs, the protocol indicated in Fig. 1 was followed. The results are provided in Table I. This protocol indicated that, following the removal of mismatches attributable to closely associated family members or mismatches detected anomalously due to repeats within a protein, 3 IEDB peptides, which were a mismatch to hg19, also overlapped the position of the TCGA mutant AA. For details of the results that were obtained by pursuing this approach, including the discounted IEDB peptides that were anomalously recovered using the Fig. 1 approach, please see SOM file no. 6 in Sait et al (5). Overall, these results indicate that mutant peptides in human cancer overlap apparent mutant peptides in the IEDB, suggesting that the AAs surrounding TCGA mutants are not fundamentally a hindrance to MHC binding. Notably, two of the proteins represented by the overlap of TCGA mutations and IEDB non-hg19 peptides represent the extracellular matrix, ITGB 3 and collagen type II $\alpha 1$, an emerging topic in the field of cancer research $(4,6,7)$.

However, the general paucity of the overlap of the two databases strongly indicates that, from a bioinformatic perspective, there is very little information available for determining which cancer drivers or passenger mutations have the potential of significant MHC binding. This conclusion is even more striking considering the extensive MHC polymorphism and protease activities that could impact binding affinities of cancer peptides (8).

In conclusion, there is a strong case to be made for the development of a more comprehensive human immuno-peptidome project, with the particular aim of determining whether cancer peptides are selected for the reduced likelihood of MHC occupancy.

\section{References}

1. He Y and Xiang Z: Databases and in silico tools for vaccine design. Methods Mol Biol 993: 115-127, 2013.

2. Helmberg W: Bioinformatic databases and resources in the public domain to aid HLA research. Tissue Antigens 80: 295-304, 2012.

3. Akbani R, Ng PK, Werner HM, Shahmoradgoli M, Zhang F, Ju Z, Liu W, Yang JY, Yoshihara K, Li J, et al: A pan-cancer proteomic perspective on the cancer genome atlas. Nat Commun 5: 3887, 2014.

4. Parry ML, Ramsamooj M and Blanck G: Big genes are big mutagen targets: A connection to cancerous, spherical cells? Cancer Lett 356: 479-482, 2015.

5. Sait S, Fawcett T and Blanck G: Supporting online materials for Overlap of The Cancer Genome Atlas and the Immune Epitope Database. http://www.universityseminarassociates. com/Supporting_online_material_for_scholarly_pubs.php.Accessed June 10, 2016.

6. Parry ML and Blanck G: Flat cells come full sphere: Are mutant cytoskeletal-related proteins oncoprotein-monsters or useful immunogens? Hum Vaccin Immunother 12: 120-123, 2016.

7. Naba A, Clauser KR, Whittaker CA, Carr SA, Tanabe KK and Hynes RO: Extracellular matrix signatures of human primary metastatic colon cancers and their metastases to liver. BMC Cancer 14: 518, 2014.

8. Cronin K, Escobar H, Szekeres K, Reyes-Vargas E, Rockwood AL, Lloyd MC, Delgado JC and Blanck G: Regulation of HLA-DR peptide occupancy by histone deacetylase inhibitors. Hum Vaccin Immunother 9: 784-789, 2013. 